\title{
The Geometrical Theory of the Structure of Nuclei, Atoms, and Molecules
}

\author{
Amagh Nduka \\ Department of Physics and Mathematics, Federal University of Technology, Owerri, Nigeria \\ Email: amaghnduka@yahoo.com.au
}

Received 23 May 2014; revised 29 June 2014; accepted 11 July 2014

Copyright (C) 2014 by author and Scientific Research Publishing Inc.

This work is licensed under the Creative Commons Attribution International License (CC BY). http://creativecommons.org/licenses/by/4.0/

c) (i) Open Access

\section{Abstract}

Quantum geometrodynamics (QGD) has established the following fundamental facts: First, every elementary particle is the physical realization of a certain irreducible 4-quantum operator of spin (rank) $0,1 / 2$ or 1 . A photon (boson) is the physical realization of an irreducible 4-quantum operator of spin zero. A fermion is the physical realization of an irreducible 4-quantum operator of spin $1 / 2$. A graviton (boson) is the physical realization of an irreducible 3-quantum operator of spin zero, and the Ws and mesons (bosons) are the physical realizations of irreducible 3-quantum operator of rank one. Second, the particles of every composite fermion system (nuclei, atoms, and molecules) reside in a certain 4-quantum space which is partitioned into an infinite set of subspaces of dimension $4^{n} \quad(n=1,2,3, \cdots, \infty ; n$ is the index of the subspace and $n$ is called principal quantum number by physicists, and period by chemists) each of which is reducible to a set of 2-level cells [1]. With these two fundamental facts, the complexities associated with atomic, nuclear, and molecular many-body problems have evaporated. As an application of the reducibility scenario we discuss in this paper the explicit construction of the periodic table of the chemical elements. In particular we show that each chemical element is characterized by a state ket $\left|E_{n} ; \ell, m_{\ell} ; s, m_{s}\right\rangle$ where $\ell$ is orbital angular momentum, $s=1 / 2, E_{\mathrm{n}}=E_{1}+k h v \quad(k=0,1,2,3, \cdots, \infty$, $E_{1}$ is the Schrödinger first energy level, and $v$ is the Lamb-Retherford frequency).

\section{Keywords}

Reducibility, Irreducibility, Periodicity, Fermion, Quantum Number, Index

\section{Introduction}

Democritus, the father of Greek Chemistry, was the originator of the atomic hypothesis. In 4C B.C. he came up 
with his atomic hypothesis that atoms are elementary indivisible and indestructible particles of which all matter was composed. The ideas of Democritus were expressed in the poem "De Rerum Natura" by Lucretius in the IC B.C.

There was, however, a rival hypothesis due to Aristotle which asserted that all matter was composed of varying proportion of the four Aristotelian elements, namely, earth, water, air, and fire. Because of the authority and overwhelming influence of Aristotle, the Democritian hypothesis was rejected, and the Aristotelian hypothesis was in force for about 2000 years.

Through the pioneering works of restless chemists of the 17, 18, and 19 centuries AD, notably Robert Boyle, Antoine Lavoisier, and John Dalton, the Aristotelian hypothesis was roundly condemned and eventually rejected. Their efforts, backed up by experimental results, helped to reinstate the Democritian hypothesis. Between 1860 and 1913 AD the empirical works of Chemists J.A.R. Newlands, D.I, Mendeleeve, H.G.J. Mosley and many others were summarized in a table called the Periodic Table of the Chemical Elements. The periodic table gave birth to the periodic law: The physical and chemical properties of the chemical elements vary periodically with the atomic number.

The periodic law implied that the atom, unlike the Democritian atom, had to be composite. The desire to determine what basic constituents of the atom could give rise to such a fundamental law brought physicists into the atomic fray. The fundamental experiments done between 1887 and 1932 by H. Hertz, A.H. Becquerel, Marie and Pierre Curie, E. Rutherford, J. Chadwick and many others brought about a new understanding of the structure and the nature of the forces in the atom. The atom consists of a central core called the nucleus surrounded by electrons, and the forces therein are non-Newtonian. By the 1920s classical field theory had made a remarkable transition to quantum field theory through the pioneering works of theorists M. Planck, L. de Broglie, W. Heisenberg, M. Born, P. Jordan, W. Pauli, E. Schrodinger, O. Klein, W. Gordon, P.A.M. Dirac, and others.

In 1926 Erwing Schrodinger took a giant step in the development of quantum mechanics with the construction of the Schrodinger equation which he applied to the Hydrogen atom. Schrodinger's theory of the hydrogen atom recorded some spectacular results, but was flawed by the fact that it was a non-relativistic theory, amongst many other defects. In 1928 P.A.M. Dirac created his relativistic theory of the hydrogen atom called the Dirac equation [2]. Dirac's theory is seminal in that it incorporated an internal degree of freedom called the spin of the electron and introduced antimatter into physics. Viewed from recent developments, Dirac's theory was the first ever formal quantium physical theory in that it was constructed entirely in terms of 4-operators, namely, $p^{\mu}, \gamma^{\mu}$, and $\mathrm{A}^{\mu}$.

As far as we know, no formal approach exists today for the treatment of many-electron atoms. The problem is so complex that only Schrodinger-based approximation methods have been developed: Variation calculations are used to obtain the ground states of the lightest atoms: for heavier atoms, the central-field approximation, Thomas-Fermi model, Hartree and Hartree-Fock approximations; and for nuclei, various many-body techniques are employed depending on the structure of the system. One major reason why these methods cannot yield satisfactory results is that, as palpably demonstrated by the Dirac's theory, atoms are embedded in a 4-dimensional pseudoeuclidean background. Further, atoms, nuclei, and molecules are discrete systems, hence differential equations are not applicable. The Schrodinger theory is therefore not expected to apply to many-electron atoms! It is for this reason that we decide to consider other methods for the treatment of many-particle atoms and nuclear systems.

\section{The Reducibility Scenario}

The situation that confronts us in many-fermion systems is similar to the problem that gave birth to statistical mechanics at the beginning of the 20th century. Like that case a completely new approach is needed here.

Our solution is based on the reducibility theorem according to which a 4-quantum subspace of index $n \geq 2\left(4^{n}=N\right.$, say $)$ is reducible according to the fundamental formula [1],

$$
N=2+\sum_{\ell=l}^{n-1} 2\left[\delta_{n n}+(2 \ell+1)\right]
$$

The " 2 " outside the summation sign in (1) comes from the subspace of index $n=1$, which is reducible to $N=4=(2+\overline{2})$-the $2(\overline{2})$ being the dimension of the subspace of index (rank) $1 / 2$. A 4-quantum space of index $1 / 2$ has no classical analogue-physically it is a 2-level cell occupied by fermions ("2") and antifer- 
mions (" $\overline{2}$ ”); the " 2 " gives the $\mathrm{He}(n=1)$ group, and “ $\overline{2}$ ” the antihelium group of chemical elements. Formula (1) is certainly true for atoms of the $\mathrm{Ne}(n=2), \operatorname{Ar}(n=3), \operatorname{Kr}(n=4), \operatorname{Xe}(n=5), \operatorname{Rn}(n=6)$, etc. groups of chemical elements as we shall see.

\section{The Periodic Table}

The Formula (1) gives the structural design for all fermion systems in the universe. The structure can be interpreted by analogy with gravitation: Each term (period) gives the "floor" of a "building" in an electrostatic environment established by the nucleus and electrons. The first period (first term) is the "ground floor", with antifermions (positrons and antineutrinos) forming the "foundation"; the second period (second term), " $(2+6)$ ", is the "first floor/roof", with the " 2 " serving as the "deck" separating the ground floor and the first floor; the third period (third term), " $(2+6+10)$ ", gives the "second floor" with the "2" again serving as the "deck" separating the first floor and the second floor-the " 10 " cannot constitute another floor in this period because there is no provision for a deck separating it and the second floor, that floor will be built in the $4^{\text {th }}$ period. The other periods are interpreted similarly. We shall now construct the periodic table of the chemical elements using the foregoing prescription and the dimensionality theorem. Here $(X(a))$ gives element " $X$ " of atomic number $z=a$.

First period $(n=1)$.

Geometrical structure: $(\overline{2}: 2)$.

This is a "foundation" structure, the "ground floor" of the atomic world built with fermions and antifermions. For the electronic world the structure is (2), the s-shell having two levels, with the electronic configuration.

$$
1 s^{2} \text {. }
$$

State Kets: $\left|1 / 2, m_{s}\right\rangle, m_{s}=-1 / 2(-),+1 / 2(+)$ where we have suppressed $E_{n}(k=0)$ from $\left|E_{1}, 1 / 2, m_{s}\right\rangle$ from here on.

Chemical elements:

$$
\left|1 / 2, m_{s}\right\rangle:-|1 / 2,-\rangle:(\mathrm{H}(1)),|1 / 2,+\rangle:(\mathrm{He}(2)) .
$$

Second period $(n=2):(2+6)$.

This is the first floor which has two levels (s-shell) as the deck and six levels (p-shell) as the first floor, with electronic configuration

$$
2 s^{2} 2 p^{6}
$$

State Kets: $\left\{\left|1 / 2, m_{s}\right\rangle,\left|1, m_{1} ; 1 / 2, m_{s}\right\rangle\right\}$.

Chemical elements:

$$
\begin{gathered}
\left|1 / 2, m_{s}\right\rangle:-|1 / 2,-\rangle:(\operatorname{Li}(3)),|1 / 2,+\rangle:(\mathrm{Be}(4)) . \\
\left|1, m_{1} ; 1 / 2, m_{s}\right\rangle:|1,-1 ; 1 / 2,-\rangle:(\mathrm{B}(5)),|1,-1,1 / 2,+\rangle:(\mathrm{C}(6)) . \\
|1,0 ; 1 / 2,-\rangle:(\mathrm{N}(7)),|1,0 ; 1 / 2,+\rangle:(\mathrm{O}(8)) . \\
|1,+1 ; 1 / 2,-\rangle:(\mathrm{F}(9)),|1,+1 ; 1 / 2,+\rangle:(\mathrm{Ne}(10)) .
\end{gathered}
$$

Third period $(n=3):(2+6+10)$.

The admissible structure here is

$$
3 s^{2} 3 p^{6}
$$

with the $3 d$-shell outstanding, as explained before.

State Kets: $\left\{\left|1 / 2, m_{s}\right\rangle,\left|1, m_{1} ; 1 / 2, m_{s}\right\rangle\right\}$.

Chemical elements:

$$
\begin{gathered}
\left|1 / 2, m_{s}\right\rangle:|1 / 2,-1\rangle:(\operatorname{Na}(11)),|1 / 2,+\rangle:(\operatorname{Mg}(12)) . \\
\left|1, m_{1} ; 1 / 2, m_{s}\right\rangle:-,|1,-1,1 / 2,-\rangle:(\operatorname{Al}(13)),|1,-1 ; 1 / 2,+\rangle:(\operatorname{Si}(14)) .
\end{gathered}
$$




$$
\begin{gathered}
|1,0,1 / 2,-\rangle:(\mathrm{P}(15)),|1,0 ; 1 / 2,+\rangle:(\mathrm{S}(16)) . \\
|1,+1 ; 1 / 2,-\rangle:(\mathrm{Cl}(17)),|1,+1,1 / 2,+\rangle:(\operatorname{Ar}(18)) .
\end{gathered}
$$

4th period $n=4: \quad(2+6+10+14)$.

Taking cognizance of the $3 d$-shell, the admissible structure is

$$
4 s^{2}\left(3 d^{10}\right) 4 p^{6}
$$

with $4 d$ and $4 f$ shells outstanding because there is no provision for "decks" for them.

State Kets: $\left\{\left|1 / 2, m_{s}\right\rangle,\left|2, m_{1} ; 1 / 2, m_{s}\right\rangle,\left|1, m_{1} ; 1 / 2, m_{s}\right\rangle\right\}$.

Chemical elements:

$$
\begin{gathered}
\left|1 / 2, m_{s}\right\rangle:-,|1 / 2,-\rangle:(\mathrm{K}(19)),|1 / 2,+\rangle:(\mathrm{Ca}(20)) . \\
\left|2, m_{1} ; 1 / 2, m_{s}\right\rangle:-,|2,-2,1 / 2,-\rangle:(\operatorname{Sc}(21)),|2,-2 ; 1 / 2,+\rangle:(\operatorname{Ti}(22)) . \\
|2,-1,1 / 2,-\rangle:(\mathrm{V}(23)),|2,-1 ; 1 / 2,+\rangle:(\operatorname{Cr}(24)) . \\
|2,0 ; 1 / 2,-\rangle:(\operatorname{Mn}(25)),|2,0 ; 1 / 2,+\rangle:(\operatorname{Fe}(26)) . \\
|2,+1 ; 1 / 2,-\rangle:(\operatorname{Co}(27)),|2,+1 ; 1 / 2,+\rangle:(\operatorname{Ni}(28)) . \\
|2,+2,1 / 2,-\rangle:(\mathrm{Cu}(29)),|2,+2 ; 1 / 2,+\rangle:(\mathrm{Zn}(30)) . \\
\left|1, m_{1} ; 1 / 2, m_{s}\right\rangle:-,|1,-1 ; 1 / 2,-\rangle:(\operatorname{Ga}(31)),|1,-1 ; 1 / 2,+\rangle:(\operatorname{Ge}(32)) . \\
|1,0 ; 1 / 2,-\rangle:(\operatorname{As}(33)),|1,0 ; 1 / 2,+\rangle:(\operatorname{Se}(34)) . \\
|1,+1 ; 1 / 2,-\rangle:(\operatorname{Br}(35)),|1,+1 ; 1 / 2,+\rangle:(\operatorname{Kr}(36)) .
\end{gathered}
$$

5th period $(n=5):(2+6+10+14+18)$.

The procedure now has a familiar ring: The admissible structure is

$$
5 s^{2}\left(4 d^{10}\right) 5 p^{6} .
$$

$4 f, 5 d, 5 f$, and $5 g$ shells are outstanding there being no decks on which these floors would stand.

State Kets: $\left\{\left|1 / 2, m_{s}\right\rangle,\left|2, m_{1}, 1 / 2, m_{s}\right\rangle,\left|1, m_{1} ; 1 / 2, m_{s}\right\rangle\right\}$.

Chemical elements:

$$
\begin{gathered}
\left|1 / 2, m_{s}\right\rangle:-,|1 / 2,-\rangle:(\operatorname{Rb}(37)),|1 / 2,+\rangle:(\operatorname{Sr}(38)) . \\
\left|2, m_{1} ; 1 / 2, m_{s}\right\rangle:-,|2,-2,1 / 2,-\rangle:(\mathrm{Y}(39)),|2,-2 ; 1 / 2,+\rangle:(\operatorname{Zr}(40)) . \\
|2,-1 ; 1 / 2,-\rangle-:(\operatorname{Nb}(41)),|2,-1 ; 1 / 2,+\rangle:(\operatorname{Mo}(42)) . \\
|2,0 ; 1 / 2,-\rangle:(\operatorname{Tc}(43)),|2,0 ; 1 / 2,+\rangle:(\operatorname{Ru}(44)) . \\
|2,+1 ; 1 / 2,-\rangle:(\operatorname{Rh}(45)),|2,+1 ; 1 / 2,+\rangle:(\operatorname{Pd}(46)) . \\
|2,+2 ; 1 / 2,-\rangle:(\operatorname{Ag}(47)),|2,+2 ; 1 / 2,+\rangle:(\operatorname{Cd}(48)) . \\
\left|1, m_{1} ; 1 / 2, m_{s}\right\rangle:-,|1,-1 ; 1 / 2,-\rangle:(\operatorname{In}(49)),|1,-1 ; 1 / 2,+\rangle:(\operatorname{Sn}(50)) . \\
|1,0 ; 1 / 2,-\rangle:(\operatorname{Sb}(51)),|1,0 ; 1 / 2,+\rangle:(\operatorname{Te}(52)) . \\
|1,+1 ; 1 / 2,-\rangle:(\mathrm{I}(53)),|1,+1 ; 1 / 2,+\rangle:(\operatorname{Xe}(54)) .
\end{gathered}
$$


6th period $(n=6):(2+6+10+14+18+22)$.

Given the deck " 2 " here "structural" stability requires that the $4 f, 5 d$, and $6 p$ shells constitute part of a floor on the $6 s$ deck. Thus, the admissible structure is

$$
6 s^{2}\left(4 f^{14} 5 d^{10}\right) 6 p^{6}
$$

with the $5 f, 5 g, 6 d, 6 f, 6 g$ and $6 h$ shells outstanding.

State Kets: $\left\{\left|1 / 2, m_{s}\right\rangle,\left|3, m_{1}, 1 / 2, m_{s}\right\rangle,\left|2, m_{1} ; 1 / 2, m_{s}\right\rangle,\left|1, m_{1} ; 1 / 2, m_{s}\right\rangle\right\}$.

Chemical elements:

$$
\begin{gathered}
\left|1 / 2, m_{s}\right\rangle:-,|1 / 2,-\rangle:(\operatorname{Cs}(55)),|1 / 2,+\rangle:(\operatorname{Ba}(56)) . \\
\left|3, m_{1} ; 1 / 2, m_{s}\right\rangle:-,|3,-3,1 / 2,-\rangle:(\operatorname{La}(57)),|3,-3 ; 1 / 2,+\rangle:(\operatorname{Ce}(58)) . \\
|3,-2 ; 1 / 2,-\rangle:(\operatorname{Pr}(59)),|3,-2 ; 1 / 2,+\rangle:(\operatorname{Nd}(60)) . \\
|3,-1 ; 1 / 2,-\rangle:(\operatorname{Pm}(61)),|3,-1 ; 1 / 2,+\rangle:(\operatorname{Sm}(62)) . \\
|3,0 ; 1 / 2,-\rangle:(\operatorname{Eu}(63)),|3,0 ; 1 / 2,+\rangle:(\operatorname{Gd}(64)) . \\
|3,+1 ; 1 / 2,-\rangle:(\operatorname{Tb}(65)),|3,+1 ; 1 / 2,+\rangle:(\operatorname{Dy}(66)) . \\
|3,+2 ; 1 / 2,-\rangle:(\operatorname{Ho}(67)),|3,+2 ; 1 / 2,+\rangle:(\operatorname{Er}(68)) . \\
|3,+3 ; 1 / 2,-\rangle:(\operatorname{Tm}(69)),|3,+3 ; 1 / 2,+\rangle:(\operatorname{Yb}(70)) . \\
\left|2, m_{1} ; 1 / 2, m_{s}\right\rangle:-,|2,-2,1 / 2,-\rangle:(\operatorname{Lu}(71)),|2,-2 ; 1 / 2,+\rangle:(\mathrm{Hf}(72)) . \\
|2,-1 ; 1 / 2,-\rangle:(\operatorname{Ta}(73)),|2,-1 ; 1 / 2,+\rangle:(\operatorname{W}(74)) . \\
|2,0 ; 1 / 2,-\rangle:(\operatorname{Re}(75)),|2,0 ; 1 / 2,+\rangle:(\operatorname{Os}(76)) . \\
|2,+1 ; 1 / 2,-\rangle:(\operatorname{Ir}(77)),|2,+1 ; 1 / 2,+\rangle:(\operatorname{Pt}(78)) . \\
|2,+2 ; 1 / 2,-\rangle:(\operatorname{Au}(79)),|2,+2 ; 1 / 2,+\rangle:(\operatorname{Hg}(80)) . \\
\left|1, m_{1} ; 1 / 2, m_{s}\right\rangle:-,|1,-1,1 / 2,-\rangle:(\mathrm{T}(81)),|1,-1 ; 1 / 2,+\rangle:(\operatorname{Pb}(82)) . \\
|1,0 ; 1 / 2,-\rangle:(\operatorname{Bi}(83)),|1,0 ; 1 / 2,+\rangle:(\operatorname{Po}(84)) . \\
|1,+1 ; 1 / 2,-\rangle:(\operatorname{At}(85)),|1,+1 ; 1 / 2,+\rangle:(\operatorname{Rn}(86)) .
\end{gathered}
$$

7th period $(n=7)(2+6+10+14+18+22+26)$.

The 7 th period is similar to the 6th period.

The structure (electronic configuration) that improves stability is

$$
7 s^{2}\left(5 f^{14} 6 d^{10}\right) 7 p^{6},
$$

with the $5 g, 6 f, 6 g, 6 h, 7 d, 7 f, 7 g, 7 h, 7 k$ outstanding.

State Kets: $\left.\left\{\left|1 / 2, m_{s}\right\rangle,\left|3, m_{1}, 1 / 2, m_{s}\right\rangle,\left|2, m_{1} ; 1 / 2, m_{s}\right\rangle|| 1, m_{1} ; 1 / 2, m_{s}\right\rangle\right\}$.

Chemical elements:

$$
\begin{gathered}
\left|1 / 2, m_{s}\right\rangle:-,|1 / 2,-\rangle:(\operatorname{Fr}(87)),|1 / 2,+\rangle:(\operatorname{Ra}(88)) . \\
\left|3, m_{1} ; 1 / 2, m_{s}\right\rangle:-,|3,-3 ; 1 / 2,-\rangle:(\operatorname{Pa}(91)),|3,-3 ; 1 / 2,+\rangle:(\mathrm{U}(92)) . \\
|3,-2 ; 1 / 2,-\rangle:(\operatorname{Np}(93)),|3,-2 ; 1 / 2,+\rangle:(\operatorname{Pu}(94)) .
\end{gathered}
$$




$$
\begin{gathered}
|3,-1 ; 1 / 2,-\rangle:(\operatorname{Am}(95)),|3,-1 ; 1 / 2,+\rangle:(\operatorname{Cm}(96)) . \\
|3,0 ; 1 / 2,-\rangle:(\operatorname{Bk}(97)),|3,0 ; 1 / 2,+\rangle:(\operatorname{Cf}(98)) . \\
|3,+1 ; 1 / 2,-\rangle:(\operatorname{Es}(99)),|3,+1 ; 1 / 2,+\rangle:(\operatorname{Fm}(100)) . \\
|3,+2 ; 1 / 2,-\rangle:(\operatorname{Md}(101)),|3,+2 ; 1 / 2,+\rangle:(\operatorname{No}(102)) . \\
|3,+3 ; 1 / 2,-\rangle:(\operatorname{Lr}(103)),|3,+3 ; 1 / 2,+\rangle:(\operatorname{Rf}(104)) . \\
\left|2, m_{1} ; 1 / 2, m_{s}\right\rangle:-,|2,-2,1 / 2,-\rangle:(\operatorname{Db}(105)),|2,-2 ; 1 / 2,+\rangle:(\operatorname{Sg}(106)) . \\
|2,-1 ; 1 / 2,-\rangle:(\operatorname{Bh}(107)),|2,-1 ; 1 / 2,+\rangle:(\operatorname{Hs}(108)) . \\
|2,0 ; 1 / 2,-\rangle:(\operatorname{Mr}(109)),|2,0 ; 1 / 2,+\rangle:(\operatorname{Uun}(110)) . \\
|2,+1 ; 1 / 2,-\rangle:(\operatorname{Uuu}(111)),|2,+1 ; 1 / 2,+\rangle:(\operatorname{Uub}(112)) . \\
|2,+2 ; 1 / 2,-\rangle:(?(113)),|2,+2 ; 1 / 2,+\rangle:(\operatorname{Fl}(114)) . \\
\left|1, m_{1} ; 1 / 2, m_{s}\right\rangle:-,|1,-1,1 / 2,-\rangle:(?(115)),|1,-1 ; 1 / 2,+\rangle:(\operatorname{Lv}(116)) . \\
|1,0 ; 1 / 2,-\rangle:(?(117)),|1,0 ; 1 / 2,+\rangle:(?(118)) . \\
|1,+1 ; 1 / 2,-\rangle:(?(119)),|1,+1 ; 1 / 2,+\rangle:(?(120)) .
\end{gathered}
$$

This procedure can in principle be continued indefinitely so that one can construct any atom one desires; but the atomic structures become progressively unstable. Actual laboratory "construction" of these unstable atoms is possible but they have only a fleeting existence.

\section{Conclusions}

We infer from the foregoing that fermions reside in definite quantum states of definite angular momentum: $(s=1 / 2)$ for the ground state and $(s=1 / 2 ; \ell)$ for all other states. Since $\ell$ takes discrete values $(\ell \geq 1)$, there are no fermion states for which $\ell=0$, or $j=\ell \pm 1 / 2$ ! This conclusion is in complete disagreement with conventional atomic and nuclear model (informal) theories. Further, because $\ell$ takes all discrete values from $\ell=1$ to $\ell=\infty$ nature admits in principle infinite number of atoms. Finally, the labeling of atoms within each multiplex defined by $\ell$ and $s$ is arbitrary; here we have labeled the atoms consistently as follows:

$$
m_{\ell}=-\ell,-\ell+1, \cdots,+\ell ; m_{s}=-1 / 2(-),+1 / 2(+) .
$$

The fermion ground state, a pure quantum state, is described geometrically by $(2: \overline{2})$, "2" for particle and “ $\overline{2}$ " for antiparticle. The physical states, consistent with the dimensionality theorem, are $\left(2 e: 2 \bar{e} ; 2 v_{e}: 2 \bar{v}_{e}\right)$ for lepton ground state and $(2 p: 2 \bar{p} ; 2 n: 2 \bar{n})$, for nucleon ground state. From these two states one builds atoms and antiatoms, consistent with the electrical neutrality of physical states [1], $\left(2 e: 2 v_{e} ; 2 p: 2 n\right)$, which gives hydrogen and its two isotopes and helium and its two isotopes, and $\left(2 \bar{e}: 2 \overline{v_{e}} ; 2 \bar{p}: 2 \bar{n}\right)$ which gives antihydrogen and its two isotopes and antihelium and its two isotopes. We call the ground state $(n=1)$ the helium group of chemical elements.

The so called first excited state $(n=2)$ is the Neon group of chemical elements. Neutrinos are absent from this state and all other states $(n>2)$ because on account of their very tiny mass they are not bound in these states [3]. There are a total of two electrons in the $2 s$-shell and six electrons in the $2 p$-shell. In the corresponding nucleus there are a total of 8 protons and 8 neutrons: 2 protons and 2 neutrons in the 2s-shell, and 6 protons and 6 neutrons in the $2 p$-shell. This gives a total of 8 chemical elements, and a number of isotopes. Other groups of chemical elements and the associated nuclei are treated similarly.

The electronic states of molecules are more complex than the atomic and nuclear states. These states can, however, be deduced from the electronic states of atoms. As an example we consider $\mathrm{H}_{\mathrm{z}}$-molecule. The state ket 
of $\mathrm{H}_{2}$ is just the tensor product of state kets of two hydrogen atoms $(=I 1 / 2 ;-->)$. The term "bond" used by chemists has no physical meaning etc.

Our theoretically derived periodic table (PT) confirms and generalizes the conventional PT of the chemical elements. The horizontal rows of elements are called periods, numbered from 1 to $\infty$, instead of from 1 to 7 . The first period has a total of 8 levels corresponding to 2 elements, 2 antielements, 2 neutrinos, and 2 antineutrinos, whereas the conventional PT has just 2 levels corresponding to just 2 elements. The second and third periods each has 8 levels (8 elements); the fourth and fifth periods each has 18 levels (18 elements); and the sixth and seventh periods each has 32 levels (32 elements), etc. The period, defined as the number of levels of a particular period, irrespective of the number of levels belonging to other earlier periods appearing in that period, is 8. This is in complete agreement with the dimensionality law.

The vertical columns define families of elements: The first 2 columns correspond to elements of angular momentum (spin) $s=1 / 2$, the last 6 columns correspond to elements of angular momentum $\ell=1$, and the 10 columns lying between the first 2 and the last 6 columns correspond to elements of angular momentum $\ell=2$. Further the 14 columns of the Rare Earths (Lanthanide Series) and Actinide Series correspond to elements of angular momentum $\ell=3$, etc. Thus, the index $n$ of the quantum space (principal quantum number, period) enumerates the groups of chemical elements and angular momentum $\ell$ enumerates the families of chemical elements.

Lastly, we call the world constructed with leptons and nucleons matter world. A world analogous with the matter world, called antimatter world, can be constructed in a similar way with antileptons and antinucleons. Thus, there exists matter—antimatter symmetry in the fermion world.

\section{References}

[1] Nduka, A. (2012) The Geometrical Theory of Science. Applied Mathematics, 3, 1598-1600. http://dx.doi.org/10.4236/am.2012.311220

[2] Berestetskii, V.B., Lifshitz, E.M. and Pithaevskii, L.P. (1971) Relativistic Quantum Theory. Pergamon Press, Oxford.

[3] Nduka, A. (2013) The Neutrino Mass. Applied Mathematics, 4, 310-313. http://dx.doi.org/10.4236/am.2013.42047 
Scientific Research Publishing (SCIRP) is one of the largest Open Access journal publishers. It is currently publishing more than 200 open access, online, peer-reviewed journals covering a wide range of academic disciplines. SCIRP serves the worldwide academic communities and contributes to the progress and application of science with its publication.

Other selected journals from SCIRP are listed as below. Submit your manuscript to us via either submit@scirp.org or Online Submission Portal.
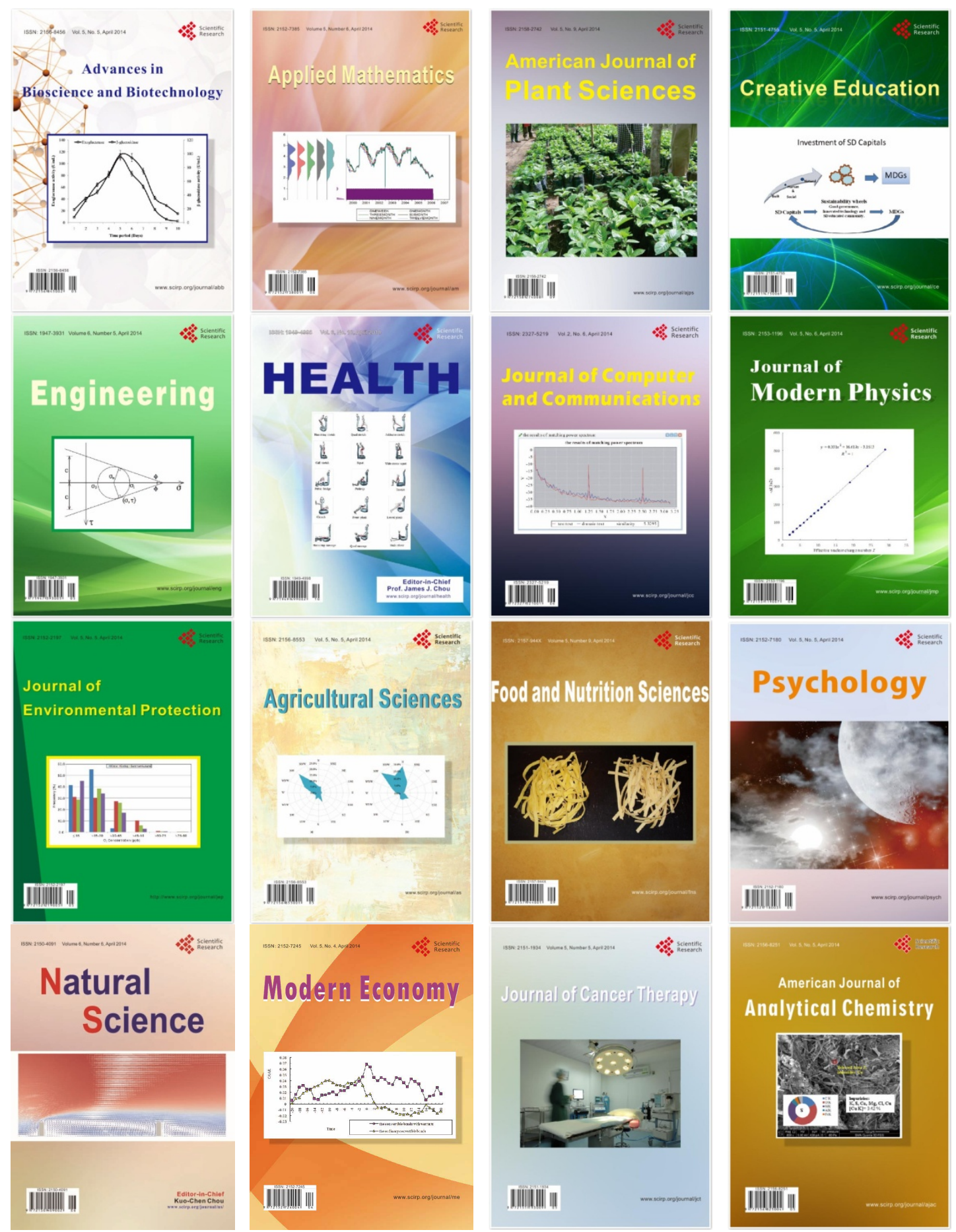